\title{
Outflow from Protostars and Angular Momentum Transfer
}

\author{
Kohji Tomisaka \\ National Astronomical Observatory, Mitaka, Tokyo 181-8588, Japan
}

\begin{abstract}
Dynamical contraction of a slowly-rotating magnetized cloud is studied using 2D magnetohydrodynamical (MHD) simulations. In the isothermal stage $\left(n \lesssim n_{\mathrm{A}} \sim 10^{10} \mathrm{~cm}^{-3}\right.$ ), the cloud evolves similarly to that expected from the Larson-Penston self-similar solution and experiences a run-away collapse. However, after the central density exceeds $\sim n_{\mathrm{A}}$, an accretion disk is formed around an adiabatic core. Just outside the core, an outflow is ejected by the effect of magnetic torque (magnetocentrifugal wind). Since $\sim 10 \%$ of the mass is ejected with almost all the angular momentum, the specific angular momentum of the protostellar core reduces to that observed in pre-main-sequence stars.
\end{abstract}

\section{Introduction}

Fragmentation of clouds and cloud cores is thought to be affected by the amount of angular momentum in them. As for angular momentum, there is the a problem that the specific angular momentum of new-born stars (e.g. T Tauri stars) $j_{*} \simeq 6 \times 10^{16}\left(R_{*} / 2 R_{\odot}\right)^{2}(P / 10 \text { day })^{-1} \mathrm{~cm}^{2} \mathrm{~s}^{-1}$ is much smaller than that of the parent molecular cloud cores $j_{\mathrm{cl}} \simeq 5 \times 10^{21}(R / 0.1 \mathrm{pc})^{2}\left(\Omega / 4 \mathrm{~km} \mathrm{~s}^{-1} \mathrm{pc}^{-1}\right) \mathrm{cm}^{2} \mathrm{~s}^{-1}$, assuming that the observed velocity gradient comes from rotational motion. Further, the specific orbital angular momentum of a binary whose separation is about $R \sim 100 \mathrm{AU}$ is equal to $j_{\text {orbit }} \simeq 4 \times 10^{19}(R / 100 \mathrm{AU})^{1 / 2}\left(M / 1 M_{\odot}\right)^{1 / 2}$ $\mathrm{cm}^{2} \mathrm{~s}^{-1}$. If the angular momentum is conserved in the star forming process, neither a binary nor single stars can be formed.

Angular momentum is reduced by the effect of magnetic fields (B-fields). Considering an oblate spheroidal cloud is threaded by poloidal B-fields parallel to the symmetry axis, the rotational motion generates torsional Alfvén waves in the intercloud medium, which propagate away from the cloud. The torsional Alfvén waves cause the intercloud medium to rotate. In this case, the angular momentum in the cloud decreases with a timescale as $t_{B} \equiv-\frac{\Omega}{\dot{\Omega}} \simeq \frac{\sigma}{2 \rho_{a} V_{A}}=$ $\frac{2 \pi G \sigma}{B_{0}}\left(4 \pi G \rho_{a}\right)^{-1 / 2}$, where $\Omega, \sigma, \rho_{a}, V_{A}$, and $B_{0}$ represent, respectively, the angular rotation speed, the column density of the cloud, the ambient density, the Alfvén speed in the ambient medium, and the magnetic field outside the cloud (Ebert et al. 1960). Since the first factor $2 \pi G \sigma / B_{0}$ is larger than unity for magnetically supercritical clouds, the timescale $t_{B}$ is comparable to the free-fall timescale in the low-density ambient medium and thus the angular momentum transfer may seem inefficient as long as the cloud contracts dynamically.

Molecular outflow begins at some epoch in the contraction stage of the cloud. The molecular outflow ejects matter and thus angular momentum. Con- 
sider the situation that $90 \%$ of the inflowing matter forms a star and the rest $(10 \%)$ is ejected by the molecular outflow. On the other hand, $0.1 \%$ of the angular momentum goes to the newly formed star and the rest is ejected by the outflow. If this occurs, the specific angular momentum of a newly formed star is reduced by a factor $10^{-3}$ from that of the parent cloud. To confirm whether this mechanism works in the process of the star formation, we have studied the evolution of a slowly rotating cloud and the formation of molecular outflows.

\section{Numerical Method \& Model}

We assume the gas is well coupled with the B-fields. The basic equations to solve are the ideal MHD equations with cylindrical symmetry and the Poisson equation for the gravitational potential. Since the gravitational collapse proceeds heavily nonhomologously, we have to cover a wide dynamic range for both the density and the size. This was obtained by using the nested grid method, in which a number of grids with different spatial resolution is prepared (here we used 24 grids from the coarsest L0 to the finest L23) and is calculated simultaneously. The finer grids are for small-scale structure near the core while the coarser ones cover the global structure.

As initial conditions, we assume a cylindrical isothermal cloud in hydrostatic balance. B-field strength is taken as proportional to $\rho^{1 / 2}$ and the cloud's rotation axis coincides with the axis of the cylinder. Density perturbations with $\lambda$ equal to that of the most unstable mode are added. Since our numerical calculations are $2 \mathrm{D}$, fragmentation and binary formtion cannot occur.

\section{Run-away Collapse Stage}

Isothermal clouds experience the run-away collapse, in which the central density increases very rapidly in a finite timescale. This is common for spherical clouds without rotation and B-fields (Larson 1969) and for a disk formed in a rotating isothermal cloud (Norman et al. 1980). The gas falling down along the B-fields forms a contracting disk (pseudo-disk) even for rotating and magnetized clouds. Contraction continues as long as the equation of state remains isothermal. In this phase, there is no evidence for an outflow (Fig.1a,c).

\section{Accretion Stage}

After the central density reaches $\rho_{\mathrm{A}} \sim 10^{10} \mathrm{~cm}^{-3}$, the thermal radiation from dust grains is trapped in the central core and an adiabatic core is formed (Larson 1969). Since the dynamical timescale of the core becomes much shorter than that of the outer isothermal envelope, the isothermal gas begins to accrete to the nearly hydrostatic adiabatic atomic core (first core). To study the evolution of this accretion stage, we use a composite polytrope model: below $\rho_{\mathrm{A}}$ the gas is assumed isothermal; for $\rho>\rho_{\mathrm{A}}$ the gas becomes adiabatic and the polytropic index $\Gamma$ is assumed as $5 / 3$ (see Tomisaka 1998).

Figure 1 illustrates the change from the isothermal run-away collapse $(\mathrm{a}, \mathrm{c})$ to the adiabatic accretion phase (b, d). Figure 1a shows that B-fields (light grey 
lines) run almost perpendicularly to the disk (dark grey surfaces) in the runaway collapse phase. Toroidal B-fields and rotational motion are not predominant (Fig.1c). On the other hand, in the accretion phase B-fields are squeezed by the effect of inflow motion in the disk and they form an hourglass-shape configuration. Further, toroidal B-fields are now formed by the rotational motions which are amplified in the accretion phase (Fig.1d). In this phase, outflow occurs (Fig.1b, d) at the distance $\simeq 50-100$ AU from the center. The time elapsed between the collapse and accretion phase is only about $1000 \mathrm{yr}$.
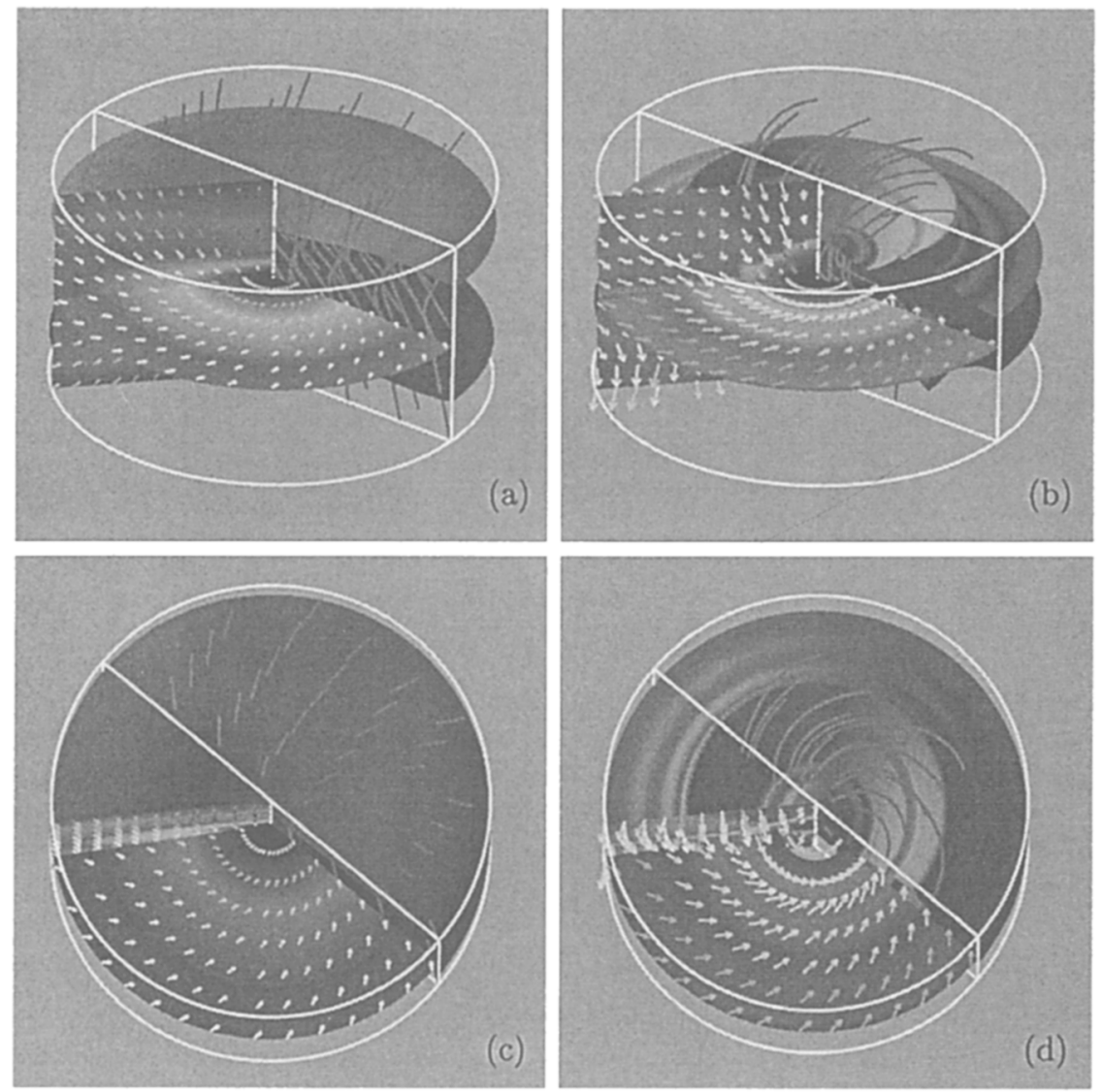

Figure 1. The left panels illustrate the structure in the run-away phase, while the right ones are for the accretion phase. The upper panels represent nearly edge-on views and the lower ones are pole-on views. These represent the structure captured by the L10 grid and the diameter of the numerical box (white circle) is equal to $\simeq 300$ AU. Isodensity surfaces and B-field lines are illustrated as well as the velocity vectors and density contours.

The Lorentz force $\mathbf{F}=(c / 4 \pi) \mathbf{j} \times \mathbf{B}$ contains the toroidal component $F_{\phi}=$ $(c / 4 \pi) \mathbf{j}_{\text {pol }} \times \mathbf{B}_{\text {pol }}$ when there exists a poloidal current $\left(\mathbf{j}_{\text {pol }}\right)$ which makes the toroidal B-fields $B_{\phi}$. Therefore, it is shown that the magnetic torque $N=r F_{\phi}$, 


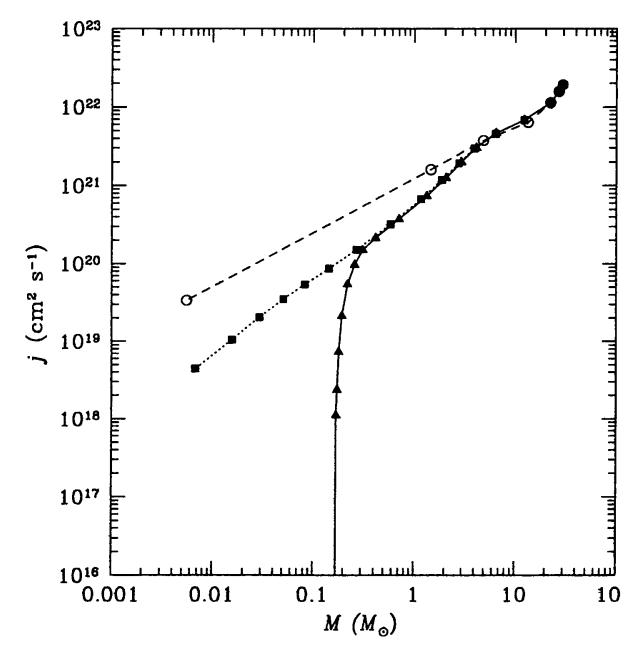

Figure 2. Specific angular momentum $j$ is plotted against the accumulated mass from the center $M$. Open circles, filled squares, and triangles denote, respectively, the beginning and the end of the runaway collapse and the accretion phase.

which changes the distribution of the angular momentum, works effectively only after the toroidal B-field is amplified by the rotational motion. The toroidal Bfields become predominant after the core formation epoch. Further, Blandford \& Payne (1982) showed that efficient angular momentum transfer from the disk to the (cold) wind material occurs only when the angle between the B-field line and the disk is smaller than $60^{\circ}$. This condition is satisfied only in the accretion phase (compare Fig.1a and.1b)

\section{Angular Momentum Transfer}

To study the effect of angular momentum transfer by the magnetic torque, the specific angular momentum is plotted against the accumulated mass from the center in Figure 2 (Tomisaka 2000). The difference between the initial stage (dashed line) and the core formation epoch (dotted line) does not come from the angular momentum transfer but from the segregation of the mass with small $j$ to form the high-density central part. True transfer occurs after the core formation. The magnetic torque increases $j$ of the matter on the disk surface and the matter with excess $j$ is ejected as the outflow, while the magnetic torque decreases $j$ inside the disk and this leads to accelerate the accretion.

That is, B-fields changes the angular momentum distribution along one field line. As a result, in $\simeq 7000 \mathrm{yr}$ after core formation $M \simeq 0.2 M_{\odot}$ is accumulated in the core and $j$ of the core becomes as small as $\sim 10^{16} \mathrm{~cm}^{2} \mathrm{~s}^{-1}$ (solid line). Therefore, it is clearly shown that the angular momentum problem of newborn stars is solved taking into account the molecular outflow driven by the magnetic torque formed just outside the first core $(R \lesssim 100 \mathrm{AU})$. 


\section{References}

Blandford, R. D., \& Payne, D. G. 1982, MNRAS, 199, 883

Ebert, R., von Hoerner, S., \& Temesvary, S. 1960, Die Entstehung von Sternen durch Kondensation diffuser Materie (Berlin: Springer), 315

Larson, R. B. 1969, MNRAS, 145, 271

Norman, M. L., Wilson, J. R., \& Barton, R. T. 1980, ApJ, 239, 968

Tomisaka, K. 1998, ApJL, 502, L163

Tomisaka, K. 2000, ApJL, 528, L41; see also

http://yso.mtk.nao.ac.jp/ tomisaka/Presentation/iau200/main/index.htm

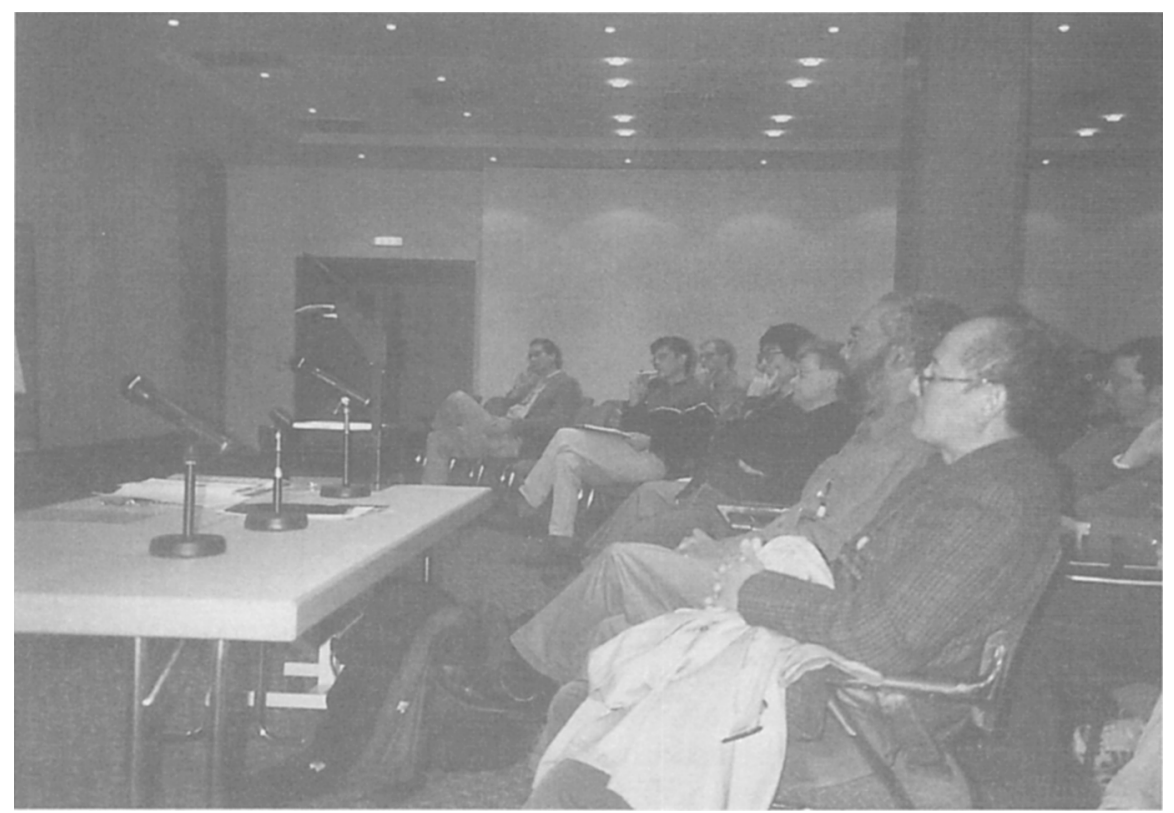

Frank Shu, Gibor Basri, Anthony Whitworth, Kohji Tomisaka, Wilhelm Kley and Alan Boss 